Article

\title{
Can Ciprofloxacin be Used for Precision Treatment of Gonorrhea in Public STD Clinics? Assessment of Ciprofloxacin Susceptibility and an Opportunity for Point-of-Care Testing
}

\author{
Johan H. Melendez ${ }^{1, *}$, Yu-Hsiang Hsieh ${ }^{2}$, Mathilda Barnes ${ }^{1}$, Justin Hardick ${ }^{1}$, \\ Elizabeth A. Gilliams ${ }^{3}$ and Charlotte A. Gaydos ${ }^{1}$ \\ 1 Division of Infectious Diseases, Johns Hopkins School of Medicine, Baltimore, MD 21205, USA; \\ mbarnes2@jhmi.edu (M.B.); jhardic1@jhmi.edu (J.H.); cgaydos@jhmi.edu (C.A.G.) \\ 2 Department of Emergency Medicine, Johns Hopkins University, Baltimore, MD 21205, USA; \\ yhsieh1@jhmi.edu \\ 3 Johns Hopkins School of Medicine, Baltimore, MD 21205, USA; Elizabeth.Gilliams@baltimorecity.gov \\ * Correspondence: jmelend3@jhmi.edu
}

Received: 18 September 2019; Accepted: 4 October 2019; Published: 14 October 2019

\begin{abstract}
Background: Given the lack of new antimicrobials to treat Neisseria gonorrhoeae (NG) infections, reusing previously recommended antimicrobials has been proposed as a strategy to control the spread of multi-drug-resistant NG. We assessed ciprofloxacin susceptibility in a large sample set of NG isolates and identified correlates associated with ciprofloxacin-resistant NG infections. Methods: NG isolates collected in Baltimore, Maryland between 2014 and 2016 were evaluated by Gyrase A (gyrA) PCR and E-test for susceptibility to ciprofloxacin. Clinical characteristics and demographics were evaluated by multivariate regression analysis to identify correlates of ciprofloxacin-resistant NG infections. Results: $510 \mathrm{NG}$ isolates from predominately African American (96.5\%), heterosexual $(85.7 \%)$, and HIV-negative $(92.5 \%)$ male subjects were included in the study. The overall percentage of isolates with mutant $g y r A$ sequences, indicative of ciprofloxacin resistance, was $32.4 \%$, and significantly increased from $24.7 \%$ in 2014 to $45.2 \%$ in 2016 ( p < 0.001$)$. Participants older than 35 years of age were 2.35 times more likely to have a gyr $A$ mutant NG infection than younger participants ( $\mathrm{p}$ 0.001). Race, sexual orientation, symptomology, or co-infection the HIV or syphilis were not associated with a particular NG gyrA genotype. Conclusions: Resistance to ciprofloxacin in Baltimore is lower than other regions and indicates that in this environment, use of ciprofloxacin may be appropriate for targeted treatment provided utilization of enhanced surveillance tools. The targeted use of ciprofloxacin may be more beneficial for individuals under 35 years of age. Point-of-care tests for NG diagnosis and susceptibility testing are urgently needed to identify individuals who can be treated with this targeted approach.
\end{abstract}

Keywords: Neisseria gonorrhoeae; gonorrhea; antimicrobial resistance; ciprofloxacin resistance; precision treatment

\section{Introduction}

Gonorrhea is the second most prevalent bacterial sexually transmitted infection (STI) worldwide, with an estimated 87 million infections in 2016 [1]. In the United States, 555,608 cases of gonorrhea were reported to the Centers for Disease Control and Prevention (CDC) in 2017, a 67\% increase from 2013 [2]. Neisseria gonorrhoeae (NG) has progressively developed resistance to all commonly-prescribed 
antimicrobials [3] and is considered as one of the top three urgent threats among antibiotic-resistant bacteria [4].

In response to the threat of multidrug-resistant NG, the World Health Organization (WHO) has proposed a global action plan, comprising several strategies, to mitigate the emergence and spread of antimicrobial resistant (AMR) NG [5]. One of the strategies proposed by the WHO to combat AMR NG is the development of molecular methodologies for monitoring and detecting antimicrobial resistance in NG. Traditionally, the determination of AMR NG has been performed by minimum inhibitory concentration (MIC) testing, which requires viable organisms for proper execution. A well developed and validated molecular method for AMR determination could obviate the requirement for a viable clinical isolate. It has been hypothesized that antimicrobial susceptibility testing (AST) at the point-of-care (POC) could lead to precision treatment, i.e., utilizing specific antibiotics based on the AMR profile of NG as opposed to syndromic management of NG infections, including reusing previously recommended antimicrobials. This approach could reduce ceftriaxone selection pressure as ceftriaxone is one of the few remaining antibiotics effective against NG, and help delay the emergence of extended spectrum cephalosporin (ESC) resistance or untreatable gonorrhea [6,7].

Ciprofloxacin, a previously recommended antimicrobial, is an excellent candidate for precision treatment as $69.9 \%$ of NG isolates collected by the gonococcal isolate surveillance project (GISP) in 2017 were susceptible to ciprofloxacin [8]. Furthermore, ciprofloxacin susceptibility can be reliably predicted through the detection of genetic markers, thus allowing for the characterization of ciprofloxacin susceptibility directly from a variety of clinical specimens, including those used for the nucleic acid amplification test (NAAT)-based detection of gonorrhea [9]. At the molecular level, resistance to ciprofloxacin is strongly associated with a single mutation at codon 91 of the gyrase $A(g y r A)$ gene [10], and detection of this mutation has been shown to be an excellent predictor of ciprofloxacin resistance $[9,10]$. Although other codons in $g y r A$, as well as in topoisomerase IV (parC), and in rare instances, penicillin binding protein 2 (PBP2/PenA) have been associated with resistance to ciprofloxacin, the vast majority of ciprofloxacin-resistant NG harbor the single mutation at codon 91 of GyrA, making this gene the target of choice for molecular screening [3,9]. As such, in 2016, a health care-based system study showed that implementation of a single molecular assay testing for gyrA mutations could be utilized in clinical care, and reduced the use of ceftriaxone while increasing the use of ciprofloxacin [11]. Furthermore, patients with wildtype gyrA NG who were treated with ciprofloxacin successfully cleared the infection at all anatomical sites of infections [12]. Despite the successful use of ciprofloxacin in clinical settings, this approach has not been evaluated in STI clinic settings, where this approach could potentially be highly effective.

Baltimore, Maryland, a large city with a high prevalence of gonorrhea (691.7/100,000 in 2017) [13], could be an excellent setting for the re-use of ciprofloxacin as a treatment option. According to our previous study, over $55 \%$ of the NG isolates collected in 2016 were susceptible to ciprofloxacin [14]. However, additional studies are necessary to better define the epidemiology of ciprofloxacin-resistant NG in Baltimore and determine whether ciprofloxacin could be effectively re-used in this particular population. In this study, we report the epidemiology of ciprofloxacin resistance in Baltimore through the molecular analysis of 510 NG isolates collected between 2014 and 2016. Additionally, clinical characteristics and demographics were evaluated to identify correlates of ciprofloxacin-resistant NG infections.

\section{Results}

A total of 510 urethral NG isolates collected from 2014 to 2016 were included in the study. The isolates were recovered from 510 male subjects (15-69 years old), predominately African American (96.5\%), heterosexual (85.7\%), and HIV-uninfected (92.5\%) (Table 1). The majority of participants $(96.7 \%)$ reported symptoms of urethritis at the time of sample of collection, and $4.3 \%$ were co-infected with syphilis. 
Table 1. Characteristics of 510 men with Neisseria gonorrhoeae infection in Baltimore, 2014-2016.

\begin{tabular}{|c|c|c|}
\hline Characteristics & Categories & Number (\%) \\
\hline & & $N=510$ \\
\hline \multirow[t]{6}{*}{ Age (Years) } & $15-19$ & $60(11.8)$ \\
\hline & $20-24$ & $117(22.9)$ \\
\hline & $25-29$ & $109(21.4)$ \\
\hline & $30-34$ & $57(11.2)$ \\
\hline & $35-44$ & $85(16.7)$ \\
\hline & $\geq 45$ & $82(16.1)$ \\
\hline \multirow[t]{4}{*}{ Race/Ethnicity } & African American & $492(96.5)$ \\
\hline & Non-Hispanic White & $11(2.2)$ \\
\hline & Hispanic & $3(0.6)$ \\
\hline & Other & $4(0.8)$ \\
\hline \multirow[t]{4}{*}{ Sexual Orientation } & Heterosexual & $437(85.7)$ \\
\hline & Bisexual & $17(3.3)$ \\
\hline & Gay & $50(9.8)$ \\
\hline & Unknown/Unspecified & $6(1.2)$ \\
\hline \multirow[t]{3}{*}{ Calendar Year } & 2014 & $170(33.3)$ \\
\hline & 2015 & $185(36.3)$ \\
\hline & 2016 & $155(30.4)$ \\
\hline \multirow[t]{10}{*}{ Symptoms } & Discharge & $459(90.0)$ \\
\hline & Dysuria & $253(49.6)$ \\
\hline & Itch in urogenital area & $19(3.7)$ \\
\hline & Lesion in urogenital area & $16(3.1)$ \\
\hline & Irritation or tingling feeling & $13(2.5)$ \\
\hline & Burning sensation & $7(1.4)$ \\
\hline & Rash & $6(1.2)$ \\
\hline & Pain in urogenital area & $5(1.0)$ \\
\hline & Other & $2(0.4)$ \\
\hline & None & $17(3.3)$ \\
\hline \multirow[t]{3}{*}{ HIV Infection } & Yes & $34(6.7)$ \\
\hline & No & $472(92.5)$ \\
\hline & Unknown & $4(0.8)$ \\
\hline \multirow[t]{2}{*}{ Concurrent Syphilis Infection } & Yes & $22(4.3)$ \\
\hline & No & $488(95.7)$ \\
\hline \multirow[t]{2}{*}{ Syphilis Diagnosis in the Past } & Yes & $25(4.9)$ \\
\hline & No & $485(95.1)$ \\
\hline \multirow[t]{2}{*}{ GyrA Genotype } & Wild type & $345(67.7)$ \\
\hline & Mutant & $165(32.4)$ \\
\hline
\end{tabular}

The number of isolates collected by year was uniformly distributed: 170 (33.3\%), 185 (36.3\%), and $155(30.4 \%)$, collected in 2014, 2015, and 2016, respectively. Genotypic typing by PCR revealed that $32.4 \%(165 / 510)$ of the isolates had mutation(s) in the gyrA gene. The percentage of isolates with gyrA mutant sequences was $24.7 \%$ (42/170), 28.7\% (53/185), and 45.2\% (70/155) in 2014, 2015, and 2016, respectively. The increase in the percentage of gyrA mutant NG from 2014 to 2016 was statistically significant $(\mathrm{p}<0.001)$ in a bivariate and multivariate regression analysis (Tables 2 and 3 ). Multivariate analysis was specifically used to determine the predictor of our outcomes adjusted for co-variates period of the $165 \mathrm{NG}$ isolates with mutant gyrA sequences; 63.6\% (105/165) were viable for susceptibility testing, and $98.1 \%$ (103/105) were confirmed as ciprofloxacin resistant by the E-test method. The remaining two isolates displayed intermediate resistance to ciprofloxacin. Susceptibility testing of 92 randomly selected isolates with gyr $A$ wildtype sequences showed that all isolates were susceptible to ciprofloxacin. 
Table 2. Bivariate analysis of association between demographic and clinical characteristics and gyr $A$ genotype among 510 men with Neisseria gonorrhoeae infection in Baltimore, 2014-2016.

\begin{tabular}{|c|c|c|c|c|}
\hline \multirow[b]{2}{*}{ Characteristics } & \multirow[b]{2}{*}{ Categories } & \multicolumn{2}{|c|}{ gyrA Genotype } & \multirow[t]{2}{*}{ p-Value } \\
\hline & & Wild Type & Mutant & \\
\hline & & $N=345$ & $N=165$ & \\
\hline \multirow[t]{3}{*}{ Age (Years)* } & $15-24$ & 135 (39.1) & $42(25.5)$ & 0.002 \\
\hline & $25-34$ & $113(32.8)$ & $53(32.1)$ & \\
\hline & $\geq 35$ & $97(28.1)$ & $70(42.4)$ & \\
\hline Race/Ethnicity & African American & $334(96.8)$ & $158(95.8)$ & 0.546 \\
\hline Sexual Orientation & Bisexual or Gay & $51(14.8)$ & $16(9.7)$ & 0.112 \\
\hline \multirow[t]{3}{*}{ Calendar Yeart } & 2014 & $128(37.1)$ & $42(25.5)$ & $<0.001$ \\
\hline & 2015 & $132(38.3)$ & $53(32.1)$ & \\
\hline & 2016 & $85(24.6)$ & $70(42.4)$ & \\
\hline Symptom-Discharge & Discharge & $312(90.4)$ & $147(89.1)$ & 0.636 \\
\hline Symptom-Dysuria & Dysuria & $176(51.0)$ & $77(46.7)$ & 0.358 \\
\hline No Symptom & Yes & $9(2.6)$ & $8(4.9)$ & 0.187 \\
\hline HIV Infection & Yes & $26(7.5)$ & $8(4.9)$ & 0.255 \\
\hline Concurrent Syphilis Infection & Yes & $15(4.4)$ & $7(4.2)$ & 0.956 \\
\hline Syphilis Diagnosis in the Past & Yes & $16(4.6)$ & $9(5.5)$ & 0.689 \\
\hline
\end{tabular}

Table 3. Multivariate regression analysis of factors associated with presence of gyrA genotype among 510 men with Neisseria gonorrhoeae infection in Baltimore, 2014-2016.

\begin{tabular}{cccc}
\hline Variables & Categories & Odds Ratio (95\% CI) & p-Value \\
\hline Age Group (years) & $15-24$ & 1.00 & \\
\hline & $25-34$ & $1.46(0.90,2.37)$ & 0.123 \\
\hline Calendar Year & Increasing each year & $1.61(1.27,2.05)$ & $<0.001$ \\
\hline
\end{tabular}

Stratification of the isolates by gyrA genotype revealed that older age was associated with a mutant gyr $A$ genotype $(\mathrm{p}=0.002)$, suggestive of an association between ciprofloxacin resistance and age (Table 2), and participants $\geq 35$ years of age were 2.35 times (95\% CI, 1.47-3.76) more likely to have a gyrA mutant NG infection than younger participants ( $<<0.001)$ (Table 3). Race, sexual orientation, symptomology, or co-infection with another STI (HIV or syphilis) were not associated with a particular NG gyrA genotype (Table 2).

\section{Discussion}

As the first step in the development of a POC test for gonorrhea diagnosis and antimicrobial susceptibility testing, we sought to determine whether ciprofloxacin could be a suitable antimicrobial for precision treatment in Baltimore. Using a molecular approach, we have shown low to moderate levels of ciprofloxacin resistance in Baltimore during a three-year period, suggesting that this antimicrobial might be a suitable option for precision treatment. Furthermore, our study identified an association between age and ciprofloxacin-resistant NG infections.

The overall percentage (32.4\%) of mutant gyrA NG infections, and thus resistance to ciprofloxacin, in our study is similar to the nationwide percentage (30.1\%) reported by GISP in 2017 [8]. The high 
percentage of wildtype gyrA (ciprofloxacin-susceptible) NG isolates in this study provides further evidence for the use of ciprofloxacin for targeted precision treatment, which may help to delay the emergence and spread of resistance to current first-line regimens [6,7]. Additionally, use of ciprofloxacin could make partner therapy easier. However, the increase in the percentage of isolates with mutated gyrA sequences from 2014 to 2016 (24.7 to $45.2 \%$ ), which is consistent with national trends [15], suggests that ciprofloxacin resistance has persisted despite ciprofloxacin not being used as a recommended treatment option for gonorrhea. Therefore, the reintroduction of ciprofloxacin as a gonorrhea treatment option will require enhanced surveillance practices to determine if and when the targeted treatment is no longer a viable option.

Our study also identified age as an important demographic correlate associated with ciprofloxacin-resistant NG infections. Men older than 35 years of age were 2.35 times more likely to have a mutant gyrA NG infection than younger men. On the contrary, men under 24 years of age were more likely to have a wildtype gyrA (ciprofloxacin-susceptible) infection. These findings suggest that younger individuals $(<24$ years of age) may be the ideal population for targeted precision treatment with ciprofloxacin. A targeted treatment approach may prove efficacious in older individuals, but caution is warranted considering the higher percentage of ciprofloxacin resistance observed in this study and the results of previous studies which have reported an association between older age and antimicrobial-resistant NG infections [16]. Contrary to previous studies [16], our study did not find an association between ciprofloxacin-resistant NG and sexual orientation; however, the number of NG isolates from men who have sex with men (MSM) was limited, and a larger study focusing on MSM may provide more details.

The re-introduction of ciprofloxacin as a treatment option could help to mitigate the emergence and spread of AMR NG. According to a modeling study, the introduction of a POC ciprofloxacin susceptibility test could help to decrease the use of ceftriaxone by as much as $66 \%$, thus potentially helping to extend the usefulness of this antimicrobial [17]. However, caution is warranted since the introduction of a POC test targeting a single antimicrobial, such as ciprofloxacin, may accelerate the emergence of triply-resistant (ciprofloxacin, ceftriaxone, and azithromycin) NG isolates [7]. These modelling studies highlight the need for the development of POC tests for resistance to multiple antimicrobials, but the complex resistance mechanisms of other antimicrobials, such as ceftriaxone [3], have hindered the development of such molecular tests. Phenotypic tests, on the other hand, may determine resistance to multiple antimicrobials, but are not currently available at the POC. Therefore, until POC tests targeting multiple antimicrobials can be developed, a single antimicrobial POC test, capable of providing an alternative treatment option, such as ciprofloxacin, might be the most suitable option. The development of a POC test for gonorrhea and identification of ciprofloxacin susceptibility is attractive because ciprofloxacin can be administered before the patient leaves the clinic.

Our study had several limitations. First, all of the isolates were collected in Baltimore, Maryland at one clinic, thus limiting the generalizability and scope of these results. However, the data reported by GISP in 2017 suggest low levels of ciprofloxacin resistance in the US [8]. Second, we had limited access to epidemiological, complete demographic, and behavioral data, which limited the scope of our analysis. It should also be noted that these samples were collected between 2014 and 2016; it is likely that the levels of ciprofloxacin susceptibility and resistance have varied since then, and these results should be viewed with that caveat. Finally, samples from women and samples from extragenital sites (pharyngeal and rectal) were not available for this analysis. Additionally, a more complete approach to the study would have been to expand molecular testing to include ParC targets associated with ciprofloxacin resistance, as well as the rare PenA target associated with ciprofloxacin resistance. However, as stated previously, the vast majority of ciprofloxacin-resistant NG harbor the single mutation at codon 91 of GyrA, and focusing on this target, as similar studies have shown, proves to be just as effective at identifying ciprofloxacin susceptibility and resistance, as expanded tests show. 


\section{Conclusions}

In conclusion, we have shown that a large proportion of the NG isolates tested in this study are susceptible to ciprofloxacin, providing further support for the use of this antimicrobial for targeted treatment if ciprofloxacin susceptibility can be determined at the POC. Current treatment guidelines for NG from multiple health organizations state that ceftriaxone (CRO) plus azithromycin (AZM), or cefixime (CFX), are the standards, but these guidelines are based on a syndromic approach to disease management [3,5]. Given the overall increase in resistance to multiple classes of antibiotics, syndromic management of NG may no longer be the most appropriate strategy. Even in this study, the increase in resistance during the study period highlights the need for continued and improved surveillance practices. Given the lower proportion of ciprofloxacin resistance in younger individuals, a targeted surveillance approach may be more beneficial for patients under 35 years of age as it increases the likelihood of identifying ciprofloxacin susceptible NG. Additional studies aimed at identifying facilitators and potential barriers towards the implementation of POC susceptibility testing and precision treatment in the STI clinic setting are warranted.

\section{Materials and Methods}

\subsection{Clinical Data}

This study was approved by the Johns Hopkins University School of Medicine Institutional Review Board. For purposes of statistical analysis to determine potential risk factors and clinical correlates associated with acquisition of ciprofloxacin-resistant NG, clinical data (age, race, sexual orientation, HIV status, current and past diagnosis of a Syphilis infection) were collected by record review.

\subsection{NG Isolates}

NG isolates were recovered from the urethra of symptomatic men seeking STI testing at the Baltimore City Health Department (BCHD) Druid Health Clinic from January 2014 to October 2016. Following culture, gram-negative diplococci, oxidase-positive bacterial isolates were presumptively classified as NG, stored in trypticase soy broth (TSB) with $20 \%$ glycerol, and frozen at $-80^{\circ} \mathrm{C}$.

For this study, isolates were recovered by growth on chocolate agar plates incubated overnight in $5 \% \mathrm{CO}_{2}$ and the colonies re-suspended in phosphate buffered saline (PBS). Two-hundred microliters of each bacterial suspension was extracted for DNA using the automated MagNA Pure LC instrument (Roche Diagnostics, Indianapolis, IN, USA). Following DNA extraction, the isolates were confirmed as NG using a previously described PCR assay targeting the opa gene $[18,19]$. For non-viable isolates, $200 \mu \mathrm{L}$ of the isolate-containing TSB media was extracted for total DNA as described above.

\subsection{Molecular Characterization of Ciprofloxacin Susceptibility}

In order to determine ciprofloxacin susceptibility at the molecular level, all isolates (viable and non-viable) were analyzed by real-time PCR for the presence/absence of mutation(s) in the gyr A gene using a previously described assay $[19,20]$. The PCR assay targets wildtype gyrA sequences, which are highly predictive of ciprofloxacin susceptibility [9]. NG isolates with negative gyrA PCR results were classified as gyrA mutant, which is indicative of ciprofloxacin resistance.

\subsection{Antimicrobial Susceptibility Testing}

All viable NG isolates with mutated gyrA sequences were further analyzed by the E-test method, (bioMérieux, Durham, NC, USA), to determine the minimum inhibitory concentration (MIC) of ciprofloxacin, as we previously described [14]. Additionally, all viable NG isolates with wildtype gyrA sequences, collected in 2016, and a subset of isolates collected in 2014 were also tested using the E-test method. Phenotypic susceptibility testing on isolates collected in 2015 was not performed, because none of those isolates were viable for analysis. Briefly, bacterial suspensions, matching a 0.5 
MacFarland standard, were plated on GC agar medium (Becton Dickinson, Sparks, MD, USA) media supplemented with 1\% IsoVitaleX (Becton Dickinson, Franklin Lakes, NJ, USA) and allowed to air dry for 5 minutes. E-test strips containing ciprofloxacin were individually placed on the inoculated agar surface according to manufacturer's recommendations, incubated at $37{ }^{\circ} \mathrm{C}$ in a moist $5 \% \mathrm{CO}_{2}$-enriched environment, and MIC results recorded after 18-24 h. The MIC was determined by reading the intercept of the inhibition zone and the E-test strip. Breakpoints for ciprofloxacin resistance were selected in accordance with guidelines set forth by the Clinical and Laboratory Standards Institute (CLSI) [21].

\subsection{Statistical Analysis}

Bivariate analysis was performed using the chi-square test. The Cochran-Armitage Trend test was performed to evaluate yearly trends. The multivariate model was used to determine the predictor of our outcomes adjusted for co-variates. In this case, age group was the key predictor, and we strongly believed that calendar years and others could be either covariates for the outcomes. Therefore, we built this full model first, then used multivariate analysis to identify the final model. The final model was built in a stepwise manner. All analyses were conducted using SAS version 9.4

Author Contributions: J.H.M., J.H., and C.A.G. contributed to the conception and design of the study. J.H.M. and J.H. contributed to analysis and interpretation of data. Y.-H.H. performed statistical analyses. E.A.G. contributed to collecting the Neisseria gonorrhoeae isolates. J.H.M. and M.B. contributed to collecting the data. J.H.M., J.H., and C.A.G drafted the manuscript. All authors reviewed and approved the final manuscript.

Funding: This research was funded by the National Institutes of Health (NIBIB 1U54 EB007958) and (NIAID U-01068613).

Conflicts of Interest: The authors declare no conflict of interest.

\section{References}

1. Rowley, J.; Vander Hoorn, S.; Korenromp, E.; Low, N.; Unemo, M.; Abu-Raddad, L.J.; Chico, R.M.; Smolak, A.; Newman, L.; Gottlieb, S.; et al. Chlamydia, gonorrhoea, trichomoniasis and syphilis: Global prevalence and incidence estimates, 2016. Bull. World Health Organ. 2019, 97, 548-562. [CrossRef] [PubMed]

2. Centers for Disease Control and Prevention. Sexually Transmitted Disease Surveillance 2017; U.S. Department of Health and Human Services: Atlanta, GA, USA, 2017. Available online: https://www.cdc.gov/std/stats17/ default.htm (accessed on 20 May 2019).

3. Unemo, M.; Del Rio, C.; Shafer, W.M. Antimicrobial Resistance Expressed by Neisseria gonorrhoeae: A Major Global Public Health Problem in the 21st Century. Microbiol. Spectr. 2016, 4, 1-10. [CrossRef] [PubMed]

4. Centers for Disease Control and Prevention. 2013. Antibiotic Resistance Threats in the United States. CDC, US Department of Health and Human Services. Available online: https://www.cdc.gov/drugresistance/pdf/ ar-threats-2013-508.pdf (accessed on 10 May 2019).

5. WHO 2012. Global Action Plan to Control the Spread and Impact of Antimicrobial Resistance in Neisseria gonorrhoeae. Available online: http://www.who.int/reproductivehealth/publications/rtis/9789241503501/en/ (accessed on 25 October 2018).

6. Sadiq, S.T.; Mazzaferri, F.; Unemo, M. Rapid accurate point-of-care tests combining diagnostics and antimicrobial resistance prediction for Neisseria gonorrhoeae and Mycoplasma genitalium. Sex. Transm. Infect. 2017, 93, S63-S68. [CrossRef] [PubMed]

7. Tuite, A.R.; Gift, T.L.; Chesson, H.W.; Hsu, K.; Salomon, J.A.; Grad, Y.H. Impact of rapid susceptibility testing and antibiotic selection strategy on the emergence and spread of antibiotic resistance in gonorrhea. J. Infect. Dis. 2017, 216, 1141-1149. [CrossRef] [PubMed]

8. Centers for Disease Control and Prevention. Gonococcal Isolate Surveillance Project (GISP) Profiles. 2017. Available online: https://www.cdc.gov/std/stats17/GISP2017/default.htm (accessed on 20 May 2019).

9. Allan-Blitz, L.T.; Wang, X.; Klausner, J.D. Wild-Type Gyrase A Genotype of Neisseria gonorrhoeae Predicts In Vitro Susceptibility to Ciprofloxacin: A Systematic Review of the Literature and Meta-Analysis. Sex. Transm. Dis. 2017, 44, 261-265. [CrossRef] [PubMed] 
10. Belland, R.J.; Morrison, S.G.; Ison, C.; Huang, W.M. Neisseria gonorrhoeae acquires mutations in analogous regions of gyrA and parC in fluoroquinolone resistant isolates. Mol. Microbiol. 1994, 14, 371-380. [CrossRef] [PubMed]

11. Allan-Blitz, L.T.; Humphries, R.M.; Hemarajata, P.; Bhatti, A.; Pandori, M.W.; Siedner, M.J.; Klausner, J.D. Implementation of a Rapid Genotypic Assay to Promote Targeted Ciprofloxacin Therapy of Neisseria gonorrhoeae in a Large Health System. Clin. Infect. Dis. 2017, 64, 1268-1270. [CrossRef] [PubMed]

12. Allan-Blitz, L.T.; Hemarajata, P.; Humphries, R.M.; Kimble, M.; Elias, S.; Klausner, J.D. Ciprofloxacin May be Efficacious in Treating Wild-Type Gyrase A Genotype Neisseria gonorrhoeae Infections. Sex. Transm. Dis. 2018, 45, e18. [CrossRef] [PubMed]

13. Center for Sexually Transmitted Infection Prevention. Division of Hygiene and Mental health. Baltimore City Health Department; Maryland Office of Planning. 2017. Available online: https://phpa.health.maryland. gov/OIDPCS/CSTIP/Pages/STI-Data-Statistics.aspx (accessed on 25 June 2018).

14. Melendez, J.H.; Hardick, J.; Barnes, M.; Page, K.R.; Gaydos, C.A. Antimicrobial Susceptibility of Neisseria gonorrhoeae Isolates in Baltimore, Maryland, 2016: The Importance of Sentinel Surveillance in the Era of Multi-Drug-Resistant Gonorrhea. Antibiotics 2018, 7, 77. [CrossRef] [PubMed]

15. St. Cyr, S.; Torrone, E.; Workowski, K. As a response to STD treatment guideline updates, have Neisseria gonorrhoeae strains regained susceptibility to ciprofloxacin? Sex. Transm. Dis. 2018, 45, S39.

16. Abraha, M.; Egli-Gany, D.; Low, N. Epidemiological, behavioural, and clinical factors associated with antimicrobial-resistant gonorrhoea: A review. F1000Research 2018, 7, 400. [CrossRef] [PubMed]

17. Turner, K.M.; Christensen, H.; Adams, E.J.; McAdams, D.; Fifer, H.; McDonnell, A.; Woodford, N. Analysis of the potential for point-of-care test to enable individualised treatment of infections caused by antimicrobial-resistant and susceptible strains of Neisseria gonorrhoeae: A modelling study. BMJ Open 2017, 7, e015447. [CrossRef] [PubMed]

18. Tabrizi, S.N.; Chen, S.; Tapsall, J.; Garlan, S.M. Evaluation of opa-based real-time PCR for detection of Neisseria gonorrhoeae. Sex. Transm. Dis. 2005, 32, 199-202. [CrossRef] [PubMed]

19. Melendez, J.H.; Hardick, J.; Barnes, M.; Barnes, P.; Geddes, C.D. Gaydos, C.A. Molecular Characterization of Markers Associated with Antimicrobial Resistance in Neisseria gonorrhoeae Identified from Residual Clinical Samples. Sex. Transm. Dis. 2018, 45, 312-315. [CrossRef] [PubMed]

20. Giles, J.; Hardick, J.; Yuenger, J.; Dan, M.; Reich, K.; Zenilman, J. Use of applied biosystems 7900HT sequence detection system and TaqMan assay for detection of quinolone-resistant Neisseria gonorrhoeae. J. Clin. Microbiol. 2004, 42, 3281-3283. [CrossRef] [PubMed]

21. Clinical and Laboratory Standard Institute. Performance Standards for Antimicrobial Susceptibility Testing, 28th ed.; CLSI supplement M100; CLSI: Wayne, PA, USA, 2018. 